\title{
DOULAS APOIANDO MULHERES DURANTE O TRABALHO DE PARTO: EXPERIÊNCIA DO HOSPITAL SOFIA FELDMAN
}

Leão MRC, Bastos MAR. Doulas apoiando mulheres durante o trabalho de parto: experiência do Hospital Sofia Feldman. Rev Latino-am Enfermagem 2001 maio; 9(3):90-4.

O apoio durante o parto, também denominado suporte intraparto pode ser realizado tanto por profissionais do serviço, enfermeiras e parteiras, como por acompanhantes leigas treinadas, denominadas doulas. Os objetivos deste artigo visam fazer uma revisão da literatura sobre suporte intraparto realizado por doulas e relatar a experiência de um hospital filantrópico de nível secundário.

UNITERMOS: trabalho de parto, enfermagem

\section{DOULAS SUPPORTING WOMEM DURING LABOR: THE EXPERIENCE OF SOFIA FELDMAN HOSPITAL}

Support during travail, also called social intratravail support, can be accomplished by hospital staff, nurses and midwives as well as by trained companions called "doulas". The purpose of this work is to conduct a literature review on labor support accomplished by "doulas" and report the experience of a secondary-level philanthropical hospital.

KEY WORDS: labor support, nursing

\section{"DOULAS" APOYANDO MUJERES DURANTE EL TRABAJO DE PARTO: EXPERIENCIA DEL HOSPITAL SOFIA FELDMAN}

El apoyo durante el parto, también denominado soporte intraparto puede ser realizado por los profesionales de servicio, enfermeras y parteras, como por los acompañantes capacitadas, llamadas "doulas". Los objetivos de éste artículo son: presentar una revisión de la literatura sobre el soporte intraparto realizadas por "doulas" y relatar las experiencias desarrolladas en un hospital filantrópico de nivel secundario.

TÉRMINOS CLAVES: trabajo de parto, enfermería

\footnotetext{
${ }^{1}$ Enfermeira Obstetra do Hospital Sofia Feldman, Mestranda em Enfermagem; ${ }^{2}$ Enfermeira, Doutora, Professor Adjunto. Escola de Enfermagem da Universidade
} Federal de Minas Gerais 
Com o processo de institucionalização e medicalização da assistência ao parto, iniciado no século XIX, através da incorporação paulatina da obstetrícia pela medicina e da difusão da atenção médica hospitalar, a tradição de familiares participarem do nascimento, principalmente as mulheres, foi sendo desconsiderada, e as mães passaram a ser atendidas nos hospitais apenas por profissionais de saúde, permanecendo sozinhas durante um longo período do trabalho de parto.

Algumas práticas que eram correntes antes da institucionalização e medicalização da assistência obstétrica e que foram descaracterizadas do processo de parturição, como a posição vertical durante o segundo estágio, o apoio constante de familiares ou amigas da mulher durante 0 trabalho de parto, a assistência domiciliar ao parto e o contato contínuo entre pais e bebês após 0 parto, passam a ser incentivadas, agora, portanto, com respaldo científico.

Dentre estas práticas, está o apoio oferecido à mulher durante o parto, temática abordada no presente artigo.

0 apoio durante o parto, também denominado suporte intraparto, pode ser realizado tanto por profissionais do serviço, enfermeiras e parteiras, como por acompanhantes leigas treinadas, denominadas doulas.

Doula é uma palavra de origem grega que significa servente, escrava. Posteriormente, foi usada para descrever aquela que assiste a mulher em casa após o parto, cozinhando para ela, ajudando a cuidar das outras crianças, segurando o bebê e tudo mais que for necessário. No contexto atual, o termo doula se refere àquela que está ao lado, que interage com, que ajuda a mulher em algum momento durante o período perinatal, seja na gravidez, no trabalho de parto ou na amamentação ${ }^{(1)}$.

Doula é uma mulher sem experiência técnica na área da saúde, que orienta e assiste a nova mãe no parto e nos cuidados com o bebê. Seu papel é segurar a mão da mulher, respirar com ela, sorrir para ela, prover encorajamento e tranqüilidade ${ }^{(2)}$.

Considerando que trata-se de uma temática nova na realidade brasileira torna-se de fundamental importância não só a revisão da literatura, como também, a avaliação das iniciativas institucionais de incorporação das doulas como suporte no trabalho de parto da sua clientela.

Diante do exposto, os objetivos deste artigo visam fazer uma revisão da literatura sobre suporte intraparto realizado por doulas e relatar a experiência de um hospital filantrópico de nível secundário.
A literatura científica é ampla em estudos que abordam o apoio social que as mulheres recebem durante o período perinatal e em especial durante $o$ trabalho de parto.

As atividades de apoio durante o trabalho de parto podem ser classificadas em 4 categorias: suporte emocional, que consiste em encorajar, tranqüilizar e estar presente continuamente; medidas de conforto físico como massagens e compressa fria; suporte de informações através de orientações, instruções e conselhos e defesa que consiste em interpretar os desejos do casal frente aos profissionais do hospital e agir em favor do mesmo ${ }^{(3)}$.

Em artigo de revisão comparou-se os vários achados na literatura científica sobre apoio durante o trabalho de parto, oferecido por profissionais de saúde, pais, familiares, amigos, monitores treinados e doulas; os autores concluem que 0 apoio oferecido por doulas produz os resultados mais extensivos e significativos, é metodologicamente seguro e produz efeitos consistentemente positivos nos resultados psicossociais e obstétricos ${ }^{(4)}$.

0 primeiro estudo que teve como objetivo investigar os efeitos da presença contínua de uma acompanhante qualificada (doula) ao lado da parturiente durante o trabalho de parto, foi realizado em 1980. Trata-se de um ensaio clínico randomizado com 136 parturientes, no Hospital da Previdência Social na Guatemala. 0 grupo experimental que recebeu apoio de doulas durante todo 0 trabalho de parto foi comparado ao grupo controle que recebeu assistência de enfermagem tradicional. Não era permitido familiares acompanharem as parturientes. As doulas eram mulheres leigas que acompanhavam a mulher da admissão ao parto, realizavam massagens nas costas, seguravam as mãos e conversavam com a mulher ${ }^{(5)}$.

Foi também observado, no grupo experimental, menor incidência de problemas perinatais, menor tempo de trabalho de parto e a observação do comportamento materno evidenciou mais interação da mãe com o bebê $\hat{~}^{(5)}$.

Em 1986, foi realizado o segundo ensaio clínico randomizado, com 465 primigestas saudáveis no Hospital da Previdência Social na Guatemala, no qual observou-se que no grupo experimental, que recebeu apoio das doulas, houve menor ocorrência de complicações perinatais incluindo parto cesárea, uso de ocitocina e bebês admitidos na unidade de cuidados intensivos neonatais ${ }^{(6)}$.

Outro estudo randomizado com 412 primigestas saudáveis, realizado em um Hospital de ensino nos Estados Unidos com práticas obstétricas modernas, onde não é permitido a permanência de acompanhantes com as parturientes, contatou-se que no grupo experimental que recebeu apoio das doulas foi observado menor taxa de anestesia peridural para parto vaginal, 
menor uso de ocitocina, menor tempo de trabalho de parto, menor taxa de cesariana, menor incidência de bebês retidos e com sepsis neonatal $^{(7)}$.

0 mecanismo pelo qual o apoio contínuo fornecido pelas doulas melhorou os resultados perinatais nestes três estudos parece estar associado à ansiedade materna. As altas taxas de catecolaminas (adrenalina e noradrenalina) liberadas na circulação sangüínea como conseqüência da intensa ansiedade materna, explicam a progressão lenta do trabalho de parto e as complicações perinatais.

Estudos realizados em mulheres e em macacas relacionaram os níveis séricos elevados de adrenalina e noradrenalina na circulação sangüínea, com a redução da atividade uterina e do fluxo sangüíneo uterino, levando a extensão do trabalho de parto e asfixia fetal ${ }^{(8-10)}$.

Em um estudo realizado em macacas grávidas, que foram submetidas a tensão psicológica, sem estimulação dolorosa e sem qualquer tipo de contato físico, foi encontrada severa asfixia fetal com diminuição do ph arterial fetal ${ }^{(11)}$.

Assim, a presença constante da doula ao lado da parturiente, tranqüilizando-a, encorajando-a e provendo conforto físico, reduz a ansiedade materna e conseqüentemente os níveis de catecolaminas circulantes. A parturiente se sente mais segura e tranqüila, o trabalho de parto transcorre mais rapidamente, necessitando de menos intervenções obstétricas. Os achados destes estudos sugerem a importância do suporte intraparto e apontam a doula como uma opção segura e eficaz.

Em 1991, realizou-se um ensaio clínico randomizado que teve como objetivo estudar o suporte intraparto realizado por doulas, em 189 nulíparas no Coronation Hospital, África do Sul, uma instituição comunitária que atende mulheres de baixa renda. Não houve diferenças estatisticamente significantes entre o grupo com suporte e o grupo controle, quanto aos parâmetros fisiológicos do progresso do trabalho de parto e quanto a necessidade de intervenções obstétricas ${ }^{(12)}$.

Porém na entrevista estruturada realizada 24 horas após o parto, as mulheres do grupo controle referiram mais ansiedade durante o trabalho de parto, relataram que se sentiram muito tensas e que o trabalho de parto foi muito difícil. Também mais mulheres do grupo controle classificaram a dor do trabalho de parto como intensa $^{(12)}$

Na sexta semana após o parto, as mulheres do grupo com suporte apresentaram maior índice de aleitamento exclusivo ao seio, reportaram mais facilidade em tornarem-se mães e bom desempenho nos cuidados com o bebê em relação as mulheres do grupo controle ${ }^{(12)}$.

Em estudo realizado no Coronation Hospital que teve como objetivo identificar a incidência de depressão pós-parto, constatouse que as mulheres que receberam suporte apresentaram uma média de escore de auto estima mais elevado e uma média de escore de ansiedade menor que as mulheres do grupo controle ${ }^{(13)}$.

Quanto à intensidade de depressão, as mulheres do grupo controle apresentaram maior proporção de depressão moderada e grave, enquanto as mulheres do grupo experimental apresentaram uma maior proporção de depressão leve ${ }^{(13)}$.

Os autores comentam que para explicar estes resultados, é necessário aceitar a premissa que o momento do trabalho de parto é de uma sensibilidade única para os fatores ambientais e que os acontecimentos e interações que ocorrem durante este período, têm conseqüências psicológicas intensas e duradouras. Poucas experiências da vida se aproximam a do nascimento em relação ao nível de stress, ansiedade, dor, esforço e desordem emocional $^{(13)}$.

Em um estudo randomizado com 724 mulheres nulíparas no Instituto de Seguros Sociais do México foi também encontrado resultados favoráveis: maior frequêencia de amamentação exclusiva ao seio um mês após o parto, menor tempo de trabalho de parto e alto grau de controle sobre a experiência do parto no grupo que recebeu apoio das doulas ${ }^{(14)}$.

0 primeiro estudo qualitativo sobre suporte social durante o trabalho de parto e nascimento, também realizado no Instituto de Seguros Sociais do México, teve como objetivo identificar o que a mulher sentia durante o trabalho de parto se acompanhada pela doula ou se deixada sozinha. 0 estudo incluiu entrevistas com as mulheres, médicos, enfermeiras e doulas e observações estruturadas das interações entre os diferentes atores (mulherdoula, mulher-profissionais do hospital e doula-profissionais do hospital ) nas enfermarias da maternidade ${ }^{(15)}$.

Os resultados publicados foram referentes às entrevistas profundas realizadas com 16 mulheres, 8 acompanhadas por doulas e 8 sem acompanhantes. Foi observado que as mulheres acompanhadas pelas doulas tiveram uma experiência de parto mais positiva. A diferença mais importante entre os dois grupos foi quanto à forma de expressar sentimentos sobre o próprio trabalho de parto, o senso de controle do processo e a auto percepção.

Os autores concluem que as atividades desenvolvidas pelas doulas são necessárias durante a assistência obstétrica para o bem estar emocional das mulheres.

\section{EXPERIÊNCIA DO HOSPITAL SOFIA FELDMAN}

0 Hospital Sofia Feldman (HSF) é uma instituição filantrópica, conveniada com o SUS, prestadora de assistência primária e secundária à saúde da criança, adolescente e mulher, que sempre permitiu aos familiares acompanhar a parturiente durante o trabalho de parto e nascimento.

0 hospital é de pequeno porte com atendimento em nível 
ambulatorial e hospitalar. Conta com 64 leitos, sendo 35 na maternidade, 21 na unidade de pediatria e 8 na unidade "mãe canguru". Em 1998 registrou-se, em média, na maternidade 280 partos por mês. 0 hospital foi reconhecido pelo UNICEF em 1995 como Amigo da Criança e funciona, desde sua inauguração, em sistema de alojamento conjunto.

Porém, mesmo sendo permitida a presença de um acompanhante com a parturiente, muitas permanecem sozinhas durante o parto sendo acompanhadas apenas pelos profissionais do serviço. Aos companheiros ou maridos é permitido o acompanhamento na sala de parto ou esporadicamente no pré parto, pois, a inexistência de leitos individualizados dificulta a presença deles ao lado da mulher durante todo o trabalho de parto.

Em 1996, uma média de 60 a 70\% das mulheres não tiveram acompanhante durante o parto. Apesar da maioria dos profissionais do hospital estarem engajados numa proposta de assistência humanizada ao parto e sentirem necessidade de dar apoio e de estarem constantemente ao lado das parturientes, as diversas funções e atividades que desenvolvem não permitem uma presença contínua ao lado da mulher.

Diante destes dados e percebendo que algumas mulheres da comunidade local, ao acompanharem familiares ou amigas em trabalho de parto, davam um importante apoio emocional para as parturientes, alguns profissionais de saúde, juntamente com a Associação Comunitária de Amigos e Usuários do Hospital Sofia Feldman (ACAU/HSF) e a direção do hospital, idealizaram o projeto "Doula Comunitária".

0 projeto foi idealizado com base na literatura científica que recomenda a introdução das doulas nas enfermarias de trabalho de $\operatorname{parto}^{(2,5-7,12-15)}$.

0 Projeto "Doula Comunitária" foi viabilizado pela ACAU/ HSF, que tem como uma de suas finalidades o melhoramento do atendimento e dos serviços prestados pelo hospital e a representação dos interesses dos usuários perante a direção da instituição. 0 projeto, através da parceria entre a associação e 0 hospital, possibilita a participação ativa da comunidade no controle social e na assistência prestada às parturientes e puérperas.

Definiu-se como doula comunitária uma senhora da comunidade recrutada pela ACAU/HSF para exercer trabalho voluntário de acompanhante da mulher durante o trabalho de parto, o parto e o puerpério, na maternidade do hospital.

As mulheres foram selecionadas pela comunidade e por uma equipe composta por assistente social, enfermeira e psicólogo. Receberam treinamento da equipe multiprofissional do hospital quanto a gestação, parto, puerpério, amamentação, cuidados com bebê, planejamento familiar, papel da doula, rotinas da instituição e comportamento ético.

As principais características observadas pelos profissionais na seleção das candidatas ao papel de doula foram:

- vivência favorável da maternidade;

- ser calma, tranqüila, carinhosa e solidária;

- ser paciente, discreta e saber ouvir;

- ter idade mínima de 21 anos;

- ter saúde física e mental;

- residir preferencialmente na comunidade assistida pelo hospital.

Os objetivos do projeto, fundamentados pela literatura científica são:

- proporcionar apoio constante à mulher e seus familiares durante o trabalho de parto, parto e puerpério, possibilitando vivências de prazer e satisfação nestes momentos tão especiais da vida;

- melhorar resultados perinatais como reduzir o tempo de trabalho de parto e o índice de complicações perinatais, evitar o uso de medicação para alívio da dor, diminuir a taxa de parto operatório e aumentar a taxa de aleitamento exclusivo ao seio;

- resguardar um tratamento individualizado e personalizado à mulher, fortalecendo-a como cidadã perante ao aparato médico institucionalizado.

Como principais atribuições da doula comunitária destacam-se:

- priorizar e acompanhar a parturiente que está sozinha sem o acompanhamento de familiares;

- dar apoio emocional a mulher em trabalho de parto, elogiando-a, tranqüilizando-a e respirando com ela;

- proporcionar conforto físico encaminhando a mulher ao banho, segurando sua mão e massageando suas costas;

- fazer os contatos com os profissionais e familiares que a mulher desejar;

- ser uma presença amigável e constante para a parturiente e seus familiares;

- incentivar a amamentação e a interação pais-bebê;

- fazer visita domiciliar se necessário.

0 trabalho das doulas teve início em junho de 1997. Já foram realizados vários treinamentos e são realizadas reuniões mensais para esclarecimento de dúvidas e discussão sobre assuntos de interesse das doulas e do serviço. As doulas são também acompanhadas e avaliadas continuamente pelos profissionais do serviço. Os profissionais são, constantemente, orientados sobre as atividades e o papel das doulas como também, sobre os objetivos do suporte oferecido por elas.

São 14 doulas, sendo uma para cada período de 12 horas. Elas recebem uma ajuda de custo referente aos gastos com alimentação e transporte. Esta ajuda de custo é amparada legalmente pela lei $n^{\circ} 9.608$ - DOU de 19/02/1998 que normatiza o serviço voluntário. Por ter apenas uma doula por turno de 12 horas, é imprescindível que as doulas priorizem acompanhar a mulher que está sem acompanhante da família e que também orientem os familiares presentes na enfermaria de pré parto quanto aos recursos 
para confortar fisicamente e emocionalmente as parturientes.

0 trabalho tem sido divulgado pela imprensa falada e escrita e está tendo uma repercussão favorável na comunidade. Após a implantação do projeto, uma média de $70 \%$ de mulheres são acompanhadas durante o trabalho de parto, por familiares ou por doulas.

\section{CONSIDERAÇÃOES FINAIS}

Embora o projeto "Doula Comunitária" seja considerado uma experiência satisfatória pelos profissionais da instituição, até 0 momento nenhum tipo de avaliação sistematizada foi realizada.

Com a finalidade de avaliar essa experiência, sugere-se a realização de estudos qualitativos com o objetivo de compreender os significados da vivência do parto para as mulheres que são acompanhadas por doulas.

Estudos de natureza qualitativa partem do pressuposto

\section{REFERÊNCIAS BIBLIOGRÁFICAS}

1. Raphael D. The tender gift breastfeeding. New York (NY): Schocken Books; 1973.

2. Nolan M. Supporting women in labour: the doula's role. Mod Midwife 1995 March; 5:12-5.

3. Hodnett ED, Osborn RJE. Effects of continuous intrapartum professional support on childbirth outcomes. Res Nurs Health 1989;12:289-97.

4. Chalmers B, Wolman W. Social support in labor: a selective review. J Psychosom Obstetr Gynaecol 1993 March; 14(1):1-15.

5. Sosa R, Kennel JH, Klaus MH, Robertson S, Urrutia J. The effects of a supportive companion on perinatal problems, length of labor, and mother-infant interaction. N Engl J Med 1980 Sept.; 303:597600.

6. Klaus MH, Kennel JH, Robertson S, Sosa R Effects of social support during parturition on maternal and infant morbidity. Br Med J1986 Sept.; 293:585.

7. Kennel JH, Klaus MH, Mcgrath S, Robertson S, Hinkley C. Continuous emocional support during labor in US Hospital. JAMA 1991 May; 265: 2197-201.

8. Zuspan FP, Cibilis LA, Pose SV. Myometrial and cardiovascular responses to alterations in plasma epinephrine and norepinephrine. Am J Obstetr Gynecol 1962 May; 84:841-51.

9. Adamsons K, Mueller-Heubach E, Meyers RE. Procuction of fetal asphia in the rhesus monkey by administration of catecholamines to the mother. Am J Obstetr Gynecol 1971; 109:248-62. de que por meio das pesquisas interpretativas podemos compreender os significados atribuídos pelos sujeitos que compartilham experiências e, por meio de sua linguagem, em relatos, chegar a compreensão de sua ação.

Assim, para dizer se o acompanhamento por doulas durante o trabalho de parto é benéfico para as mulheres, é necessário saber o que ele significa para elas. É preciso permitir que as mulheres falem sobre suas vivências, sobre crenças, mitos, valores e tabus compartilhados.

0 ser humano revela a realidade por meio da linguagem oral ou escrita e pela interpretação da linguagem o pesquisador chega aos aspectos significativos essenciais para a compreensão da experiência humana ${ }^{(16)}$.

Acreditamos que estudos desta natureza, contribuirão para uma reflexão da prática dos profissionais de saúde, com vistas na melhoria da assistência que prestamos as mulheres, a partir da compreensão de suas reais necessidades e especificidades, durante estes importantes momentos de suas vidas.

10. Lederman RP, Lederman E, Work BA, Mccann DS. The relationsship of maternal anxiety, plasma catecholamines and plasma cortisol to progress in labor. Am J Obstetr Gynecol 1978; 132:495500.

11. Myers RE. Maternal psychological stress and fetal asphyxia: a study in the monkey. Am J Obstetr Gynecol 1975; 122:47-59.

12. Hofmeyer GJ, Nikodem VC, Wolman W, Chalmers B, Kramer T. Companionship to modify the clinical birth environment: effects on progress and perceptions of labour, and breastfeeding. Br I Obstetr Gynaecol 1991 Aug.; 98:756-64.

13. Wolman W, Chalmers B, Hofmeyer J, Nikodem VC. Postpartum depression and companionship in the clinical birth environment: a randomized, controlled study. Am J Obstetr Gynecol 1993 May; 168:1388-93.

14. Langer A, Campero L, Garcia C. Effects of a psychosocial supportduring labor and childbirth on breastfeeding, medical interventions and mothers 'wellbeing in a Mexican public hospital: a randomized clinical trial. Br J Obstetr Gynecol 1998 0ct; 105:105663.

15. Campero L, Garcia C, Díaz C, Ortiz O, Reynoso S, Langer A. Alone, I wouldn't have known what to do: a qualitative study on social support during labor and delivery in Mexico. Soc Sci Med 1998; 47(3): 395403.

16. Jesus MCP, Peixoto MRB, Cunha MHF. 0 paradigma heumenêutico como fundamentação das pesquisas etnográficas e fenomenológicas. Rev Latino-am Enfermagem 1998; 6(2):29-35. 